\title{
RESULTANT OPTIMIZATION OF THE THREE-DIMENSIONAL INTERSECTION PROBLEM
}

\author{
J. L. Awange ${ }^{1}$ and Y. Fukuda ${ }^{2}$ \\ ${ }^{1}$ Western Australia Centre for Geodesy, Curtin University of Technology, Perth, \\ Australia \\ ${ }^{2}$ Department of Geophysics, Kyoto University, Kitashirakawa Oiwake-cho, \\ Sakyo-ku, Kyoto City, Kyoto 606-8502, Japan.
}

\begin{abstract}
Resultant approach is here employed to optimize the dimensionless space angles to solve in a closed form the over-determined three-dimensional intersection problem. The advantages of the resultant optimization approach are the non-requirement of the approximate initial starting values, non iterative and does not rely on linearization during its operation, save for the nonlinear variance-covariancelerror propagation to generate the weight matrix. Resultant method, a branch of abstract algebra, is employed to compute the combinatorial scatters, which are then optimized to offer a closed form solution. Using the test network Stuttgart Central as an example, it is demonstrated that the resultant optimization approach can be applied as an alternative approach to conventional methods such as least squares for point positioning within the over-determined intersection framework, especially when the approximate starting values for linearization and iterative approaches are not known as may happen in Photogrammetry, Machine Vision or in Robotics.
\end{abstract}

KEYWORDS: Optimisation. Over-determination. 3D Intersection. Scatter.

\section{INTRODUCTION}

In an earlier paper [1], the closed form solution of the planar lateration and intersection problems was presented. This idea was further developed in [7],[8] to include the minimal three-dimensional intersection problem, a classical Photogrammetric positioning technique. The presence of Global Positioning System (GPS: Global Problem $\underline{\text { Solver}) ~ d e m a n d s ~ t h a t ~ g e o d e t i c ~ a n d ~ p h o t o g r a m m e t r i c ~ d i r e c t i o n ~ o b s e r v a t i o n s ~}$ (Machine Vision, "Total Observing Stations") have to be analysed in a three-dimensional Euclidean Space. The pair of tools called "Resection and Intersection" has to operate three-dimensionally. In [7],[8], we have already demonstrated how the three-dimensional intersection problem can be solved using the dimensionless space angles in the minimal sense (i.e, non-over-determined case). In practise however, one is often faced with a situation where more observations than unknowns exist thus necessitating the need to solve the over-determined problem. More often, the over-determined solutions adopted in practice are iterative in nature and thus pegging their functionality on the choice of approximate starting values, linearization for nonlinear models and iterating to achieve convergence.

The present contribution proposes the use of the resultant approach suggested by $B$. Strumfels [16],[17] as the computing engine of the Gauss-Jacobi combinatorial algorithm, see i.e., [2], to solve the nonlinear over-determined three-dimensional intersection problem. Space angles are here used in an over-determined sense to solve via resultant optimization the over-determined three-dimensional intersection problem.

The problem of initial starting values for iterative approaches has been addressed in the works of [18],[19] which proposes faster methods to find the approximate starting values. The requirements of some starting values, iterations and linearization 
however still underpin classical procedures in spite of the faster procedures for determining starting values proposed by [18], [19]. The algebraic resultant optimization that is applied in the present contribution however enjoys the advantage that all the requirements listed above for non-algebraic approaches are immaterial. The nonlinear problem is solved in an exact form with linearization permitted only during the formation of the nonlinear variance-covariancelerror propagation to generate the weight matrix of the combinatorial scatter. No starting values, linearization of the observation equations, iterations and convergence conditions are required. The only requirement is to be able to solve in a closed (exact) form systems of nonlinear equations, a condition already presented in [8] and the fulfilment of Theorem 2.1 in [4]. Other added advantages of the proposed approach are its capability to diagnose outlying observations [2] and the provision of an independent approach that can be used to control the non-algebraic classical procedures. For other works related to the treatment of nonlinear problems, the reader is referred to [9], [10], [11], [12], [13], [15], and [17].

The present contribution therefore is an extension of the closed form solution of three-dimensional intersection problem [7],[8] by employing the Gauss-Jacobi combinatorial approach presented in [2] to solve without initial starting values, linearization or iteration, the over-determined three-dimensional intersection problem.

\section{THREE-DIMENSIONAL INTERSECTION WITH MORE OBSERVATIONS THAN UNKNOWNS}

For the complete theory of three-dimensional intersection problem and the solution in closed form using resultant approach, we refer to [8]. Here the over-determined version is introduced. In order to formulate the over-determined three-dimensional intersection problem, one needs to understand the minimal version of the problem which is presented as follows; Given three space angles $\left\{\psi_{12}, \psi_{23}, \psi_{31}\right\}$ obtained from the spherical coordinates of type horizontal directions $T_{i}$ and vertical directions $B_{i}$, for $i=1, \ldots, 3$ in Figure (1), the distances $\left\{x_{1}=S_{1}, x_{2}=S_{2}, x_{3}=S_{3}\right\}$ from the unknown point $P \in \mathbb{E}^{3}$ to three other known stations $P_{i} \in \mathbb{E}^{3} \mid i=1,2,3$ have to be determined in the first step. In the second step the derived distances from step 1 are treated as pseudo-observations. From an unknown point $P \in \mathbb{E}^{3}$ to a minimum of three known points $P_{i} \in \mathbb{E}^{3} \mid i=1,2,3$ (see, e.g., Figure 1), the position $\{X, Y, Z\}$ of the unknown point $P \in \mathbb{E}^{3}$ has to be determined. When only three known stations are used to determine the three-dimensional position of the unknown station, the problem reduces to that of minimal $3 d$ closed form solution. The algebraic form of the problem (i.e., following Theorem 2.1 in [4]) was developed by first converting the nonlinear system of equations into polynomial in [8] as follows:

From Figure 1, the nonlinear system of equations for the three dimensional 3-point positioning was given as

$$
\begin{aligned}
& x_{2}^{2}=x_{1}^{2}+S_{12}^{2}-2 S_{12} \cos \left(\psi_{12}\right) x_{1} \\
& x_{3}^{2}=x_{2}^{2}+S_{23}^{2}-2 S_{23} \cos \left(\psi_{23}\right) x_{2} \\
& x_{1}^{2}=x_{3}^{2}+S_{31}^{2}-2 S_{31} \cos \left(\psi_{31}\right) x_{3}
\end{aligned}
$$

and expressed in algebraic form (2) without bilinear terms as

$$
\begin{aligned}
& x_{1}^{2}-2 S_{12} \cos \left(\psi_{12}\right) x_{1}-x_{2}^{2}+S_{12}^{2}=0 \\
& x_{2}^{2}-2 S_{23} \cos \left(\psi_{23}\right) x_{2}-x_{3}^{2}+S_{23}^{2}=0 \\
& x_{3}^{2}-2 S_{31} \cos \left(\psi_{31}\right) x_{3}-x_{1}^{2}+S_{31}^{2}=0,
\end{aligned}
$$

$\left\{x_{1}=S_{1}, x_{2}=S_{2}, x_{3}=S_{3}\right\}$ being the distances from $P_{1}$ to $P_{0}, P_{2}$ to $P_{0}$, and $P_{3}$ to $P_{0}$ respectively. 
From the formulation of the minimal case, the over-determined version of the problem can now be said to be when more than three stations are given, i.e., $P_{1}, P_{2}, P_{3}, \ldots, P_{n}$. In this case, the observations will comprise distances from $P_{1}$ to $P_{0}$, $P_{2}$ to $P_{0}, P_{3}$ to $P_{0}, \ldots, P_{n}$ to $P_{0}$ with the unknowns being $\{X, Y, Z\}$ and $n>3$. Such is the kind of problem requiring resultant optimisation herein proposed.

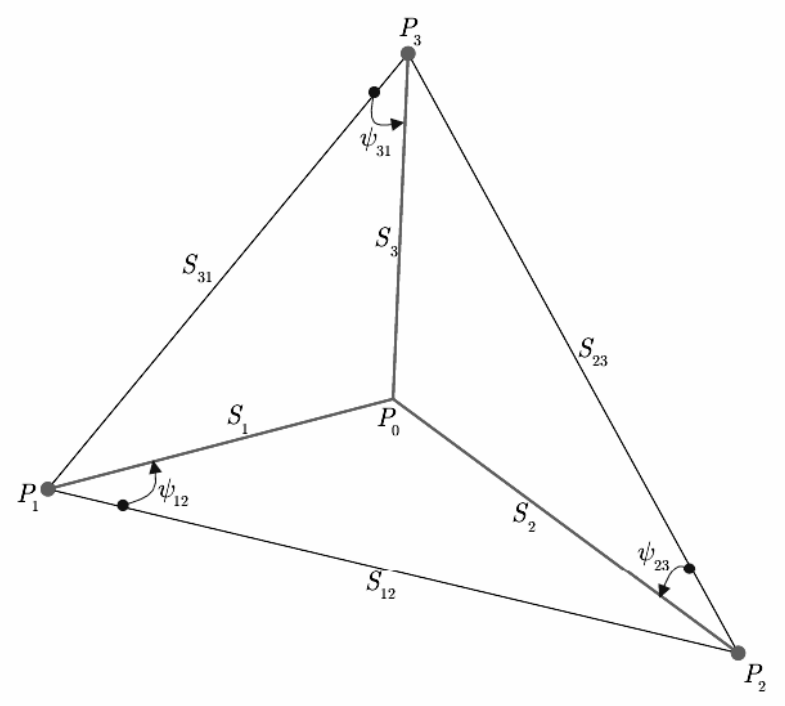

Fig. 1: 3-point intersection.

\section{RESULTANTS OPTIMISATION}

\section{Resultant approach}

Whereas the resultant of two polynomials is well known and algorithms for computing it are well incorporated into computer algebra packages such as Maple, the Multipolynomial resultant, i.e., the resultant of more than two polynomials still remain an active area of research. The resultant of two polynomials, also known as the Sylvester resultant to solve Engineering problems is exemplified in the works of [13, pp.72-76] and [3].

Here, the term resultant refers to the resultants of more than two polynomials, also called Multipolynomial resultant [14]. It is treated as a tool besides the Groebner bases to eliminate variables in solution of polynomial systems of equations. The use of resultant approach to solve in a closed form the minimum three-dimensional resection problem has already been presented in the work of $[8]$.

\section{Optimization of the three-dimensional intersection problem}

In order to solve the over-determined three-dimensional intersection problem in closed form, the resultant approach is first applied to solve for the distances $\left\{x_{1}=S_{1}, x_{2}=S_{2}, x_{3}=S_{3}\right\}$ from (2) as discussed in [8, equations 3-3 to 3-14] to give the computing engine of the combinatorial algorithm [2] as

$$
\left[\begin{array}{l}
x_{1}:=d_{4} x_{1}^{4}+d_{3} x_{1}^{3}+d_{2} x_{1}^{2}+d_{1} x_{1}+d_{0}=0 \\
x_{2}:=e_{4} x_{2}^{4}+e_{3} x_{2}^{3}+e_{2} x_{2}^{2}+e_{1} x_{2}+e_{0}=0 \\
x_{3}:=f_{4} x_{3}^{4}+f_{3} x_{3}^{3}+f_{2} x_{3}^{2}+f_{1} x_{3}+f_{0}=0
\end{array}\right.
$$


with the coefficients as already given in [8, appendix]. Using (3) and steps 1-5 of the Gauss-Jacobi combinatorial algorithm [2], the adjusted distances of the stations involved in the optimization are determined as follows:

Step 1: Given an over-determined system with $n$ observations in $m$ unknown distances, form from the $n$ observations the

$$
k(\text { noof combinations })=\frac{n !}{m !(n-m) !}
$$

minimal combinations that comprise $m$ distance equations that are to be solved using (3).

Step 2: Solve each set of $m$ distance equations from Step 1 above for distances using resultant approach derived computing engine (i.e. Eq (3)).

Step 3: Perform the nonlinear error/variance-covariance propagation to obtain the variance-covariance matrix of pseudo-observations resulting from the computational engine in Step 2.

Step 4: Using the pseudo-observations of Step 2 and the variance-covariance matrix from Step 3, adjust the pseudo-observations via the special linear Gauss-Markov model.

Step 5: Compute the adjusted distances of the network.

Once the distances have been obtained, they are used once again in the Gauss-Jacobi combinatorial algorithm to give the position. In order to compute the point position from the adjusted distances, the resultant approach is used to derive the position equations [5, Box 3-5, equations 3-35 and 3-39] as

$$
X=\frac{\left\{\left(b_{02} c_{12}-b_{12} c_{02}\right) Z+b_{12} f_{02}-b_{02} f_{12}\right\}}{\left(b_{02} a_{12}-b_{12} a_{02}\right)}
$$

and

$$
Y=\frac{\left\{\left(a_{12} c_{02}-a_{02} c_{12}\right) Z+a_{12} f_{02}-a_{02} f_{12}\right\}}{\left(a_{02} b_{12}-a_{12} b_{02}\right)}
$$

with the coefficient and the solution procedures as discussed in [5]. Equations (5) and (6) are then used as the computing engine in the Gauss-Jacobi combinatorial algorithm with steps 1-5 performed as follow:

Step 1: Given an over-determined system with $n$ observations in $m$ unknown positions, form using (4) and the $n$ observations $k$ minimal combinations that comprise $m$ position equations that are to be solved in closed form.

Step 2: Solve each set of $m$ position equations from Step 1 above for positions $\{X, Y, Z\}$ using resultant approach derived computing engine (i.e. Eqs. (5) and (6)) as discussed in [5].

Step 3: Perform the nonlinear error/variance-covariance propagation to obtain the variance-covariance matrix of pseudo-observations resulting from the computational engine in Step 2.

Step 4: Using the pseudo-observations of Step 2 and the variance-covariance matrix from Step 3, adjust the pseudo-observations via the special linear Gauss-Markov model.

Step 5: Compute the adjusted barycentric position $\{X, Y, Z\}$ of the unknown point in the network. 


\section{TEST NETWORK}

\section{Experiment}

The three-dimension position of the unknown point $K_{1}$ are to be determined by the procedure outlined above. Due to the availability of superfluous observations, made possible by the availability of several known points of the test network "Stuttgart Central" (Figure 2), the closed form three-dimensional intersection procedure gives way to procedures for solving the over-determined three-dimension intersection problem. In this case therefore, all the known GPS network stations (Haussmanstr., Eduardpfeiffer, Lindenmuseum, Liederhalle, Dach LVM, Dach FH, and Schlossplatz) of this network "Stuttgart Central" have to be used.

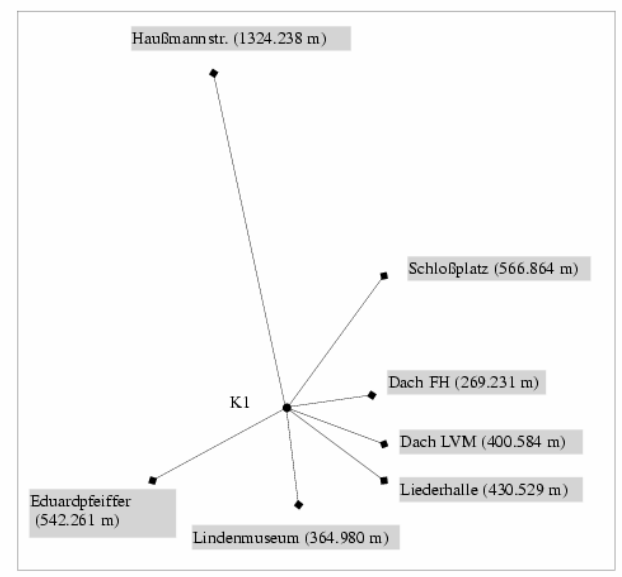

Fig. 2: Test network Stuttgart Central

The over-determined three-dimensional intersection problem is optimised using the resultant approach in six steps as follows:

Step 1: (construction of minimal combinatorial subsets for determination of network distances):

From Figure 2, 35 minimal combinatorials are formed for the network "Stuttgart Central" and for each minimal combinatorial simplex, the distances are computed from the univariate polynomials (3). Each combinatorial minimal subset results in 3 distances thus giving rise to a total of $(3 \times 35) 105$ scattered distances. The computed scattered distances $S_{i}$ link the known points $P_{i} \mid i=1, \ldots, n$ to the unknown point $P\left(K_{1}\right)$.

Step 2: (nonlinear error propagation to determine the dispersion matrix $\boldsymbol{\Sigma}$ ):

In this step, the dispersion matrix $\boldsymbol{\Sigma}$ is sought. This is achieved via the nonlinear error propagation law/variance-covariance propagation for each of the combinatorial sets $j=1, \ldots, 35$ above. The closed form observational equations for the first combinatorial subset $j=1$ are written algebraically as in (1) where $S_{i j} \mid i, j \in\{1,2,3\}, i \neq j$ are the distances between known GPS stations of the test network "Stuttgart Central", $x_{k} \mid k \in\{1,2,3\}$ are the unknown distances from the unknown GPS point $P \in \mathbb{E}^{3}$ to the known GPS stations $P_{i} \in \mathbb{E}^{3} \mid i=1,2,3$ and $\psi_{i j} \mid i, j \in\{1,2,3\}, i \neq j$ are the space angles derived from the observable of type horizontal and vertical directions to the unknown point $P \in \mathbb{E}^{3}$ from the known GPS stations $P_{i} \in \mathbb{E}^{3} \mid i=1,2,3$ respectively. With [4, Eq. 8], the Jacobi matrices are given as 


$$
\mathbf{J}_{x}=\left[\begin{array}{lll}
\frac{\partial f_{1}}{\partial x_{1}} & \frac{\partial f_{1}}{\partial x_{2}} & \frac{\partial f_{1}}{\partial x_{3}} \\
\frac{\partial f_{2}}{\partial x_{1}} & \frac{\partial f_{2}}{\partial x_{2}} & \frac{\partial f_{2}}{\partial x_{3}} \\
\frac{\partial f_{3}}{\partial x_{1}} & \frac{\partial f_{3}}{\partial x_{2}} & \frac{\partial f_{3}}{\partial x_{3}}
\end{array}\right]
$$

and

$$
\mathbf{J}_{y}=\left[\begin{array}{llllll}
\frac{\partial f_{1}}{\partial S_{12}} & \frac{\partial f_{1}}{\partial S_{23}} & \frac{\partial f_{1}}{\partial S_{31}} & \frac{\partial f_{1}}{\partial \psi_{12}} & \frac{\partial f_{1}}{\partial \psi_{23}} & \frac{\partial f_{1}}{\partial \psi_{31}} \\
\frac{\partial f_{2}}{\partial S_{12}} & \frac{\partial f_{2}}{\partial S_{23}} & \frac{\partial f_{2}}{\partial S_{31}} & \frac{\partial f_{2}}{\partial \psi_{12}} & \frac{\partial f_{2}}{\partial \psi_{23}} & \frac{\partial f_{2}}{\partial \psi_{31}} \\
\frac{\partial f_{3}}{\partial S_{12}} & \frac{\partial f_{3}}{\partial S_{23}} & \frac{\partial f_{3}}{\partial S_{31}} & \frac{\partial f_{3}}{\partial \psi_{12}} & \frac{\partial f_{3}}{\partial \psi_{23}} & \frac{\partial f_{3}}{\partial \psi_{31}}
\end{array}\right] .
$$

The values $\left\{x_{1}, x_{2}, x_{3}\right\}$ appearing in the Jacobi matrix $\mathbf{J}_{x}$ are obtained from the closed form solution of the first combinatorial set in step 1 and the partial derivative of the space angles $\psi_{i j} \mid i, j \in\{1,2,3\}, i \neq j$ derived from the observable of type horizontal and vertical directions.

Step 3: (rigorous adjustment of the combinatorial solution points in a polyhedron): Once the 105 combinatorial solution points in a polyhedron have been obtained in step 1, they are finally adjusted using the linear Gauss-Markov model [4, Eq. 15], with the dispersion matrix $\Sigma$ obtained via the error propagation law or variance-covariance propagation in step 2. Expressing each of the 105 scattered distances as

$$
S_{i}^{j}=S_{i}+\varepsilon_{i}^{j} \mid i \in\{1,2,3,4,5,6,7\}, j=\{1,2,3,4,5,6,7, \ldots, 35\},
$$

and placing the scattered distances $S_{i}^{j}$ in the vector of observation $\mathbf{y}$, the coefficients of the unknown seven distances $S_{i}$ of the network forming the coefficient matrix $\mathbf{A}$ and $\boldsymbol{\xi}$ comprising the vector of unknowns $S_{i}$, the adjusted solution is obtained via [4, Eq. 15] and the dispersion of the estimated parameters through [4 Eq. 16].

Step 4: From the 7 adjusted distances in step 3 above, 35 positional combinatorials are formed using (4) and solved for $\{X, Y, Z\}$ in close form using (5) and (6) as discussed in [5].

Step 5: (error propagation to determine the dispersion matrix $\Sigma$ ):

The variance-covariance matrix is computed for each of the combinatorial sets $j=1, \ldots, 35$ using error propagation as discussed in [6, step 2, pp.392-393].

Step 6: (rigorous adjustment of the combinatorial solution points in a polyhedron):

For each of the 35 computed coordinates of point $K_{1}$ in Figure 2 in step 2, the observation equations are written as

$$
\left[\begin{array}{l}
X^{j}=X+\varepsilon_{X}^{J} \mid, J \in\{1, \ldots, 35\} \\
Y^{j}=Y+\varepsilon_{Y}^{J} \mid, J \in\{1, \ldots, 35\} \\
Z^{j}=Z+\varepsilon_{Z}^{J} \mid, J \in\{1, \ldots, 35\} .
\end{array}\right.
$$

With the values $\left\{X^{j}, Y^{j}, Z^{j}\right\}$ treated as pseudo-observation and placed in the vector of observation $\mathbf{y}$, the coefficients of the unknown position $\{X, Y, Z\}$ being placed in the coefficient matrix $\mathbf{A}$ and $\boldsymbol{\xi}$ comprising the vector of unknowns $\{X, Y, Z\}$. The 
solution is obtained via [4, Eq. 15] and the dispersion of the estimated parameters through [4, Eq. 16].

\section{Example}

Using the data of [4, Table 1] and the observational space angles for the network [e.g., 8], the computed position of point $K_{1}$ in the experiment using the resultant optimization approach are given in Table 1 with the deviations of the resultant optimization solutions from the true GPS value given in Table 2.

In Figures 3 the deviation of the computed scatter of the distance Haussmanstr.- $K_{1}$ around their adjusted values are presented, while in Figure 4, the plot of the adjusted distances biases from those derived from GPS coordinates are plotted. The numbers in the $\mathrm{X}$-axis of Figure 4 represent distances as follows; Haussmanstr.-K1 (1), Schlossplatz-K1 (2), Dach FH-K1 (3), Dach LVM-K1 (4), Liederhalle-K1 (5), Lindenmuseum-K1 (6) and Eduardpfeiffer-K1 (7). Figure 5 indicates the plot of the combinatorial scatter in position $\{\bullet\}$ around the adjusted (barycentric) values $\{*\}$.

Table 1: Position of station K1 computed by Gauss-Jacobi combinatorial algorithm

\begin{tabular}{|l|l|l|l|l|l|l|}
\hline $\begin{array}{l}\text { Exp } \\
\text { No. }\end{array}$ & $X(m)$ & $Y(m)$ & $Z(m)$ & $\sigma_{X}(m)$ & $\sigma_{Y}(m)$ & $\sigma_{Z}(m)$ \\
\hline 1 & 4157066.1121 & 671429.6694 & 4774879.3697 & 0.00005 & 0.00001 & 0.00005 \\
\hline
\end{tabular}

Table 2: Deviation of the computed position of K1 from the real measured GPS value

\begin{tabular}{|c|c|c|c|}
\hline $\begin{array}{c}\text { Exp. } \\
\text { No. }\end{array}$ & $\Delta X(m)$ & $\Delta Y(m)$ & $\Delta Z(m)$ \\
\hline & -0.0005 & -0.0039 & 0.0007 \\
\hline
\end{tabular}

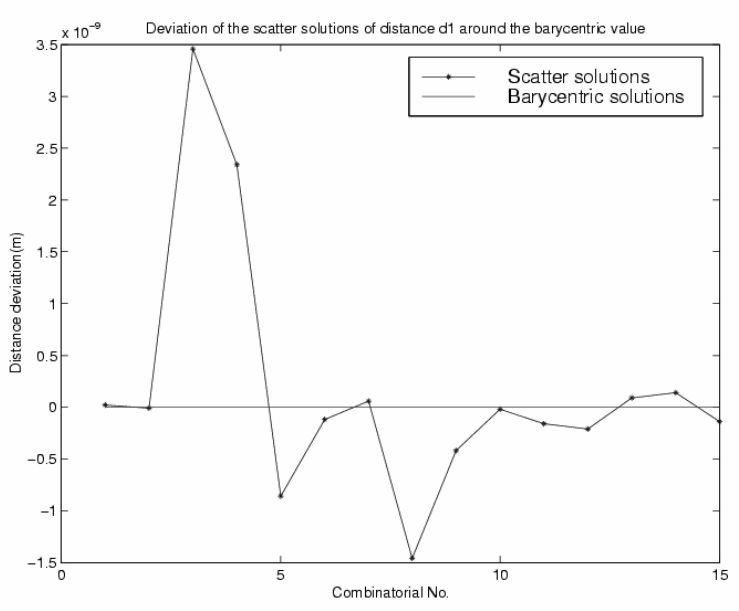

Fig. 3: Deviation of the scatter solutions of the distance Haussmanstr.-K1 from barycentric value

\section{CONCLUSION}

For problems that require the solution of over-determined three-dimensional intersection, and whose initial starting values may not be known such as in Photogrammetry, the resultant optimization offers an alternative approach provided the full information of the underlying observations are taken into consideration via the nonlinear variance-covariance/error propagation. The advantage of the resultant 
optimization in solving this problem is that no starting values, linearization or iterations is required as is the case with other procedures. Outlying combinations and observations are also identifiable. With high processing computers currently available, the issue of many combinatorials formed as a result of large observations is immaterial nor is the computing time. Routines are written that repeatedly execute the desired task once the statement that executes the combination has been written.

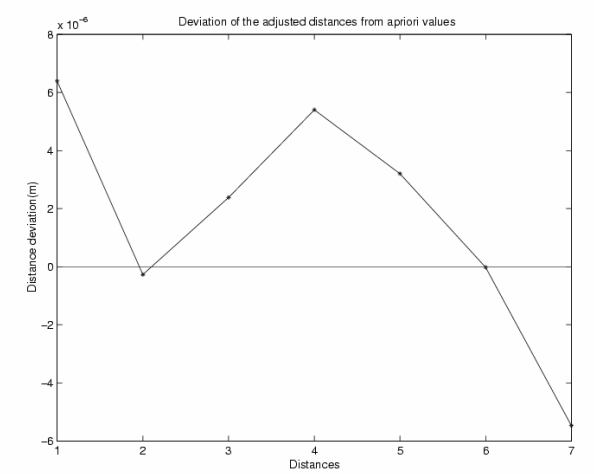

Fig. 4: Deviation of the 7-adjusted distances from real measured values

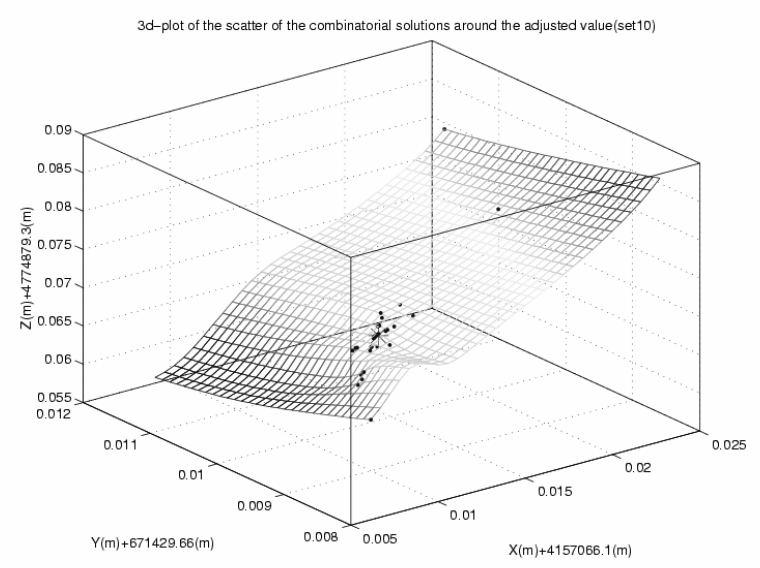

Fig. 5: Scatter of combinatorial solutions

\section{References}

1. Awange, J. L., 2003. Buchberger algorithm applied to planar lateration and intersection problems. Survey Review, 37 (290): 319-329.

2. Awange, J. L., 2005. Diagnosis of Outlier of type Multipath in GPS Pseudo-range observations. Survey Review, 38 (297): 177-189.

3. Awange, J. L. and Grafarend E., 2002. Sylvester resultant solution of the planar ranging problem. $A V N, 109$ (4): 143-146

4. Awange, J. L. and Grafarend E., 2003. Explicit solution of the over-determined three-dimensional resection problem. Journal of Geodesy, 76: 605-616.

5. Awange, J. L., Grafarend, E., Fukuda, Y., I. Ateya and Takemoto, S., 2003. Direct polynomial approach to nonlinear distance (ranging) problems. Earth, Planets and 
Space, 55: 231-241.

6. Awange, J. L., Fukuda, Y., Takemoto, S., and Grafarend, E., 2003. Ranging algebraically with more observations than unknowns. Earth, Planets and Space, 55: 387-394.

7. Awange, J. L., Grafarend, E., and Fukuda, Y., 2003. Closed form solution of the triple three-dimensional intersection problem. Zeitschrift für Geodaesie, Geoinformation und Landmanagement, 128: 395-402.

8. Awange, J. L., Fukuda, Y., Takemoto, S., and Grafarend, E., submitted. Resultants approach to the triple three-dimensional intersection problem. J. Geod. Soc. Japan.

9. Grafarend, E. and Schaffrin, B., 1989. The geometry of nonlinear adjustment-the planar trisection problem-, in Festschrift to T. Krarup, edited by E. Kejlso, K. Poder and C. C. Tscherning, pp. 149-172, Denmark.

10. Grafarend, E. and Schaffrin, B., 1991. The planar trisection problem and the impact of curvature on non-linear least -squares estimation. Computational statistics \& data analysis, 12: 187-199.

11. Guolin, L., 2000. Nonlinear curvature measures of strength and nonlinear diagnosis. Allgemeine Vermessungs-Nachrichten, 107: 109-111.

12. Krarup, T., 1982. Nonlinear adjustment and curvature, in; Forty years of thought, Delft, pp. 145-159.

13. Lohse, P., 1994. Ausgleichungsrechnung in nichtlinearen Modellen. DGK, Reihe C, Heft Nr. 429.

14. Manocha, D., and Canny, J., 1993. Multipolynomial resultant algorithms. Journal of Symbolic Computations, 5: 99-122.

15. Mautz, R., 2001. Zur Lösung nichtlinearer Ausgleichungsprobleme bei der Bestimmung von Frequenzen in Zeitreihen. DGK, Reihen C, Nr. 532.

16. Sturmfels, B., 1998. Introduction to resultants. Proceedings of Symposia in Applied Mathematics, 53: 25-39.

17. Teunissen, P., 1990. Nonlinear least squares. Manuscripta Geodaetica 15, pp. 137-150.

18. Xu, P., 2002. A hybrid global optimization method: the one-dimensional case. Journal of Computation and Applied mathematics, 147: 301-314.

19. Xu, P., 2003. A hybrid global optimization method: the multi-dimensional case. Journal of Computation and Applied mathematics, 155: 423-446. 